\title{
PROPERTIES OF OPERATIONS FOR FUZZY SOFT SETS OVER FULLY UP-SEMIGROUPS
}

\author{
AKARACHAI SATIRAD AND AIYARED IAMPAN* \\ Department of Mathematics, School of Science, University of Phayao, Phayao 56000, Thailand \\ *Corresponding author: aiyared.ia@up.ac.th

\begin{abstract}
The aim of this manuscript is to apply distributivity laws of several fuzzy sets for any fuzzy sets and study distributivity laws with any fuzzy soft sets. We investigate properties of some operations for fuzzy soft sets over fully UP-semigroups and their interrelation with respect to different operations such as "(restricted) union", "(extended) intersection", "AND", and "OR".
\end{abstract}

\section{Introduction and Preliminaries}

Several researches introduced a new class of algebras related to logical algebras and semigroups such as: In 1993, Jun et al. [7] introduced the notion of BCI-semigroups. In 2018, Iampan [6] introduced the notion of fully UP-semigroups.

In 1999, to solve complicated problems in economics, engineering, and environment, we cannot successfully use classical methods because of various uncertainties typical for those problems. Uncertainties cannot be handled using traditional mathematical tools but may be dealt with using a wide range of existing theories such as the probability theory, the theory of (intuitionistic) fuzzy sets, the theory of vague sets, the theory of interval mathematics, and the theory of rough sets. However, all of these theories have their own difficulties which are pointed out in [11]. In 2001, Maji et al. [10] introduced the concept of fuzzy soft sets as a generalization of the standard soft sets, and presented an application of fuzzy soft sets in a decision making

Received 2019-06-02; accepted 2019-07-11; published 2019-09-02.

2010 Mathematics Subject Classification. 03G25; 08A72.

Key words and phrases. fully UP-semigroup; fuzzy soft set; (restricted) union; (extended) intersection; AND; OR.

This work was supported by the Unit of Excellence, University of Phayao.

(C) 2019 Authors retain the copyrights of their papers, and all open access articles are distributed under the terms of the Creative Commons Attribution License. 
problem. In 2010, Jun et al. [8] applied fuzzy soft set for dealing with several kinds of theories in BCK/BCIalgebras. The notions of fuzzy soft BCK/BCI-algebras, (closed) fuzzy soft ideals and fuzzy soft p-ideals are introduced, and related properties are investigated. In 2013, Rehman et al. [13] studied some operations of fuzzy soft sets and give fundamental properties of fuzzy soft sets. They discuss properties of fuzzy soft sets and their interrelation with respect to different operations such as union, intersection, restricted union and extended intersection. Then, they illustrate properties of AND and OR operations by giving counter examples. Also we prove that certain De Morgan's laws hold in fuzzy soft set theory with respect to different operations on fuzzy soft sets. In 2019, Satirad and Iampan [16] introduced ten types of fuzzy soft sets over fully UP-semigroups, and investigate the algebraic properties of fuzzy soft sets under the operations of (extended) intersection and (restricted) union.

Before we begin our study, we will give the definition of a UP-algebra.

Definition 1.1. [5] An algebra $A=(A, \cdot, 0)$ of type $(2,0)$ is called a UP-algebra where $A$ is a nonempty set, $\cdot$ is a binary operation on $A$, and 0 is a fixed element of $A$ (i.e., a nullary operation) if it satisfies the following axioms:

(UP-1): $(\forall x, y, z \in A)((y \cdot z) \cdot((x \cdot y) \cdot(x \cdot z))=0)$,

(UP-2): $(\forall x \in A)(0 \cdot x=x)$,

(UP-3): $(\forall x \in A)(x \cdot 0=0)$, and

(UP-4): $(\forall x, y \in A)(x \cdot y=0, y \cdot x=0 \Rightarrow x=y)$.

From [5], we know that the notion of UP-algebras is a generalization of KU-algebras (see [12]).

On a UP-algebra $A=(A, \cdot, 0)$, we define a binary relation $\leq$ on $A[5]$ as follows:

$$
(\forall x, y \in A)(x \leq y \Leftrightarrow x \cdot y=0)
$$

Example 1.1. [18] Let $X$ be a universal set and let $\Omega \in \mathcal{P}(X)$ where $\mathcal{P}(X)$ means the power set of $X$. Let $\mathcal{P}_{\Omega}(X)=\{A \in \mathcal{P}(X) \mid \Omega \subseteq A\}$. Define a binary operation $\cdot$ on $\mathcal{P}_{\Omega}(X)$ by putting $A \cdot B=B \cap\left(A^{C} \cup \Omega\right)$ for all $A, B \in \mathcal{P}_{\Omega}(X)$ where $A^{C}$ means the complement of a subset $A$. Then $\left(\mathcal{P}_{\Omega}(X), \cdot, \Omega\right)$ is a UP-algebra and we shall call it the generalized power UP-algebra of type 1 with respect to $\Omega$. Let $\mathcal{P}^{\Omega}(X)=\{A \in \mathcal{P}(X) \mid A \subseteq \Omega\}$. Define a binary operation $*$ on $\mathcal{P}^{\Omega}(X)$ by putting $A * B=B \cup\left(A^{C} \cap \Omega\right)$ for all $A, B \in \mathcal{P}^{\Omega}(X)$. Then $\left(\mathcal{P}^{\Omega}(X), *, \Omega\right)$ is a UP-algebra and we shall call it the generalized power UP-algebra of type 2 with respect to $\Omega$. In particular, $(\mathcal{P}(X), \cdot \emptyset)$ is a UP-algebra and we shall call it the power UP-algebra of type 1 , and $(\mathcal{P}(X), *, X)$ is a UP-algebra and we shall call it the power UP-algebra of type 2. 
Example 1.2. [3] Let $\mathbb{N}$ be the set of all natural numbers with two binary operations $\circ$ and $\bullet$ defined by

$$
(\forall x, y \in \mathbb{N})\left(x \circ y=\left\{\begin{array}{ll}
y & \text { if } x<y \\
0 & \text { otherwise }
\end{array}\right)\right.
$$

and

$$
(\forall x, y \in \mathbb{N})\left(x \bullet y=\left\{\begin{array}{ll}
y & \text { if } x>y \text { or } x=0 \\
0 & \text { otherwise }
\end{array}\right) .\right.
$$

Then $(\mathbb{N}, \circ, 0)$ and $(\mathbb{N}, \bullet, 0)$ are UP-algebras.

For more examples of UP-algebras, see $[2,6,17,18]$.

In a UP-algebra $A=(A, \cdot, 0)$, the following assertions are valid (see $[5,6])$.

$$
\begin{aligned}
& (\forall x \in A)(x \cdot x=0), \\
& (\forall x, y, z \in A)(x \cdot y=0, y \cdot z=0 \Rightarrow x \cdot z=0), \\
& (\forall x, y, z \in A)(x \cdot y=0 \Rightarrow(z \cdot x) \cdot(z \cdot y)=0), \\
& (\forall x, y, z \in A)(x \cdot y=0 \Rightarrow(y \cdot z) \cdot(x \cdot z)=0), \\
& (\forall x, y \in A)(x \cdot(y \cdot x)=0), \\
& (\forall x, y \in A)((y \cdot x) \cdot x=0 \Leftrightarrow x=y \cdot x), \\
& (\forall x, y \in A)(x \cdot(y \cdot y)=0), \\
& (\forall a, x, y, z \in A)((x \cdot(y \cdot z)) \cdot(x \cdot((a \cdot y) \cdot(a \cdot z)))=0), \\
& (\forall a, x, y, z \in A)((((a \cdot x) \cdot(a \cdot y)) \cdot z) \cdot((x \cdot y) \cdot z)=0), \\
& (\forall x, y, z \in A)(((x \cdot y) \cdot z) \cdot(y \cdot z)=0), \\
& (\forall x, y, z \in A)(x \cdot y=0 \Rightarrow x \cdot(z \cdot y)=0), \\
& (\forall x, y, z \in A)(((x \cdot y) \cdot z) \cdot(x \cdot(y \cdot z))=0), \text { and } \\
& (\forall a, x, y, z \in A)(((x \cdot y) \cdot z) \cdot(y \cdot(a \cdot z))=0) .
\end{aligned}
$$

Definition 1.2. [6] Let $A$ be a nonempty set, - and $*$ are binary operations on $A$, and 0 is a fixed element of $A$ (i.e., a nullary operation). An algebra $A=(A, \cdot, *, 0)$ of type $(2,2,0)$ in which $(A, \cdot, 0)$ is a UP-algebra and $(A, *)$ is a semigroup is called a fully UP-semigroup (in short, an $f$-UP-semigroup) if the operation "*" is distributive (on both sides) over the operation ".". 
Definition 1.3. [20] A fuzzy set $\mathrm{F}$ in a nonempty set $U$ (or a fuzzy subset of $U$ ) is described by its membership function $\mathrm{f}_{\mathrm{F}}$. To every point $x \in U$, this function associates a real number $\mathrm{f}_{\mathrm{F}}(x)$ in the interval $[0,1]$. The number $\mathrm{f}_{\mathrm{F}}(x)$ is interpreted for the point as a degree of belonging $x$ to the fuzzy set $\mathrm{F}$, that is,

$$
\mathrm{F}:=\left\{\left(x, \mathrm{f}_{\mathrm{F}}(x)\right) \mid x \in U\right\}
$$

We say that a fuzzy set $\mathrm{F}$ in $U$ is constant if its membership function $\mathrm{f}_{\mathrm{F}}$ is constant.

Rosenfeld [14] introduced the notion of fuzzy subsemigroups (resp., fuzzy ideals) of semigroups as follows:

Definition 1.4. A fuzzy set $\mathrm{F}$ in a semigroup $A=(A, *)$ is called

(1) a fuzzy subsemigroup of $A$ if $(\forall x, y \in A)\left(\mathrm{f}_{\mathrm{F}}(x * y) \geq \min \left\{\mathrm{f}_{\mathrm{F}}(x), \mathrm{f}_{\mathrm{F}}(y)\right\}\right)$.

(2) a fuzzy ideal of $A$ if $(\forall x, y \in A)\left(\mathrm{f}_{\mathrm{F}}(x * y) \geq \max \left\{\mathrm{f}_{\mathrm{F}}(x), \mathrm{f}_{\mathrm{F}}(y)\right\}\right)$.

Clearly, a fuzzy ideal is a fuzzy subsemigroup.

Definition 1.5. [9] Let $\left\{\mathrm{F}_{i}\right\}_{i \in I}$ be a nonempty family of fuzzy sets in a nonempty set $U$ where $I$ is an arbitrary index set. The intersection of $\mathrm{F}_{i}$, denoted by $\bigcap_{i \in I} \mathrm{~F}_{i}$, is described by its membership function $\mathrm{f}_{\bigcap_{i \in I}} \mathrm{~F}_{i}$ which defined as follows:

$$
(\forall x \in U)\left(\mathrm{f}_{\bigcap_{i \in I} \mathrm{~F}_{i}}(x)=\inf \left\{\mathrm{f}_{\mathrm{F} i}(x)\right\}_{i \in I}\right) .
$$

The union of $\mathrm{F}_{i}$, denoted by $\bigcup_{i \in I} \mathrm{~F}_{i}$, is described by its membership function $\mathrm{f}_{\cup_{i \in I}} \mathrm{~F}_{i}$ which defined as follows:

$$
(\forall x \in U)\left(\mathrm{f}_{\cup_{i \in I} F_{i}}(x)=\sup \left\{\mathrm{f}_{\mathrm{F} i}(x)\right\}_{i \in I}\right)
$$

Theorem 1.1. Let $\mathrm{F}_{i}$ and $\mathrm{F}$ be fuzzy sets in a nonempty set $X$ where $I$ is a nonempty set. Then the following properties hold:

(1) $\mathrm{F} \cap\left(\bigcup_{i \in I} \mathrm{~F}_{i}\right)=\bigcup_{i \in I}\left(\mathrm{~F} \cap \mathrm{F}_{i}\right)$,

(2) $\left(\bigcup_{i \in I} \mathrm{~F}_{i}\right) \cap \mathrm{F}=\bigcup_{i \in I}\left(\mathrm{~F}_{i} \cap \mathrm{F}\right)$,

(3) $\mathrm{F} \cup\left(\bigcap_{i \in I} \mathrm{~F}_{i}\right)=\bigcap_{i \in I}\left(\mathrm{~F} \cup \mathrm{F}_{i}\right)$, and

(4) $\left(\bigcap_{i \in I} \mathrm{~F}_{i}\right) \cup \mathrm{F}=\bigcap_{i \in I}\left(\mathrm{~F}_{i} \cup \mathrm{F}\right)$.

Proof. Let $x \in X$. (1) First, we investigate left hand side of the equality. Assume that $\bigcup_{i \in I} \mathrm{~F}_{i}=\mathrm{F}^{\cup}$. Then $\mathrm{F} \cap\left(\bigcup_{i \in I} \mathrm{~F}_{i}\right)=\mathrm{F} \cap \mathrm{F}^{\cup}$. Also,

$$
\begin{aligned}
\mathrm{f}_{\mathrm{F} \cap F}(x) & =\min \left\{\mathrm{f}_{\mathrm{F}(x)}, \mathrm{f}_{\mathrm{F} \cup}(x)\right\} \\
& =\min \left\{\mathrm{f}_{\mathrm{F}(x)}, \mathrm{f}_{\cup_{i \in I}} \mathrm{~F}_{i}(x)\right\} \\
& =\min \left\{\mathrm{f}_{\mathrm{F}(x)}, \sup \left\{\mathrm{f}_{\mathrm{F} i}(x)\right\}_{i \in I}\right\} .
\end{aligned}
$$


Consider the right hand side of the equality. Assume that $\mathrm{F} \cap \mathrm{F}_{i}=\mathrm{F}_{i}^{\cap}$ for all $i \in I$. Then

$$
\begin{aligned}
\mathrm{f}_{\cup_{i \in I} \mathrm{~F}_{i}^{\cap}}(x) & =\sup \left\{\mathrm{f}_{\mathrm{F}_{i}^{\cap}}(x)\right\}_{i \in I} \\
& =\sup \left\{\mathrm{f}_{\mathrm{F} \cap \mathrm{F}_{i}}(x)\right\}_{i \in I} \\
& =\sup \left\{\min \left\{\mathrm{f}_{\mathrm{F}}(x), \mathrm{f}_{\mathrm{F}_{i}}(x)\right\}\right\}_{i \in I}
\end{aligned}
$$

It is clear that $\min \left\{\mathrm{f}_{\mathrm{F}(x)}, \sup \left\{\mathrm{f}_{\mathrm{F} i}(x)\right\}_{i \in I}\right\}=\sup \left\{\min \left\{\mathrm{f}_{\mathrm{F}}(x), \mathrm{f}_{\mathrm{F}_{i}}(x)\right\}\right\}_{i \in I}$. Therefore, $\mathrm{F} \cap\left(\bigcup_{i \in I} \mathrm{~F}_{i}\right)=\bigcup_{i \in I}(\mathrm{~F} \cap$ $\left.\mathrm{F}_{i}\right)$

(2) By using techniques as in (1), then (2) can be derived.

(3) First, we investigate left hand side of the equality. Assume that $\bigcap_{i \in I} \mathrm{~F}_{i}=\mathrm{F}^{\cap}$. Then $\mathrm{F} \cup\left(\bigcap_{i \in I} \mathrm{~F}_{i}\right)=$ $\mathrm{F} \cup \mathrm{F}^{\cap}$. Also,

$$
\begin{aligned}
\mathrm{f}_{\mathrm{F} \cup \mathrm{F}^{\cap}}(x) & =\max \left\{\mathrm{f}_{\mathrm{F}(x)}, \mathrm{f}_{\mathrm{F} \cap}(x)\right\} \\
& =\max \left\{\mathrm{f}_{\mathrm{F}(x)}, \mathrm{f}_{\bigcap_{i \in I} \mathrm{~F}_{i}}(x)\right\} \\
& =\max \left\{\mathrm{f}_{\mathrm{F}(x)}, \inf \left\{\mathrm{f}_{\mathrm{F} i}(x)\right\}_{i \in I}\right\} .
\end{aligned}
$$

Consider the right hand side of the equality. Assume that $\mathrm{F} \cup \mathrm{F}_{i}=\mathrm{F}_{i}^{\cup}$ for all $i \in I$. Then

$$
\begin{aligned}
\mathrm{f}_{\bigcap_{i \in I} \mathrm{~F}_{i}^{\cup}}(x) & =\inf \left\{\mathrm{f}_{\mathrm{F}_{i}^{\cup}}(x)\right\}_{i \in I} \\
& =\inf \left\{\mathrm{f}_{\mathrm{F}} \mathrm{F}_{i}(x)\right\}_{i \in I} \\
& =\inf \left\{\max \left\{\mathrm{f}_{\mathrm{F}}(x), \mathrm{f}_{\mathrm{F}_{i}}(x)\right\}\right\}_{i \in I} .
\end{aligned}
$$

It is clear that $\max \left\{\mathrm{f}_{\mathrm{F}(x)}, \inf \left\{\mathrm{f}_{\mathrm{F} i}(x)\right\}_{i \in I}\right\}=\inf \left\{\max \left\{\mathrm{f}_{\mathrm{F}}(x), \mathrm{f}_{\mathrm{F}_{i}}(x)\right\}\right\}_{i \in I}$. Therefore, $\mathrm{F} \cup\left(\bigcap_{i \in I} \mathrm{~F}_{i}\right)=\bigcap_{i \in I}(\mathrm{~F} \cup$ $\left.\mathrm{F}_{i}\right)$.

(4) By using techniques as in (3), then (4) can be derived.

Somjanta et al. [19], Guntasow et al. [4], and Satirad and Iampan [16] introduced the notion of fuzzy UP-subalgebras (resp., fuzzy near UP-filters, fuzzy UP-filters, fuzzy UP-ideals, fuzzy strongly UP-ideals) of UP-algebras as follows:

Definition 1.6. A fuzzy set $\mathrm{F}$ in a UP-algebra $A=(A, \cdot, 0)$ is called

(1) a fuzzy UP-subalgebra of $A$ if $(\forall x, y \in A)\left(\mathrm{f}_{\mathrm{F}}(x \cdot y) \geq \min \left\{\mathrm{f}_{\mathrm{F}}(x), \mathrm{f}_{\mathrm{F}}(y)\right\}\right)$.

(2) a fuzzy near UP-filter of $A$ if

(i) $(\forall x \in A)\left(\mathrm{f}_{\mathrm{F}}(0) \geq \mathrm{f}_{\mathrm{F}}(x)\right)$, and

(ii) $(\forall x, y \in A)\left(\mathrm{f}_{\mathrm{F}}(x \cdot y) \geq \mathrm{f}_{\mathrm{F}}(y)\right)$.

(3) a fuzzy UP-filter of $A$ if

(i) $(\forall x \in A)\left(\mathrm{f}_{\mathrm{F}}(0) \geq \mathrm{f}_{\mathrm{F}}(x)\right)$, and 
(ii) $(\forall x, y \in A)\left(\mathrm{f}_{\mathrm{F}}(y) \geq \min \left\{\mathrm{f}_{\mathrm{F}}(x \cdot y), \mathrm{f}_{\mathrm{F}}(x)\right\}\right)$.

(4) a fuzzy UP-ideal of $A$ if

(i) $(\forall x \in A)\left(\mathrm{f}_{\mathrm{F}}(0) \geq \mathrm{f}_{\mathrm{F}}(x)\right)$, and

(ii) $(\forall x, y, z \in A)\left(\mathrm{f}_{\mathrm{F}}(x \cdot z) \geq \min \left\{\mathrm{f}_{\mathrm{F}}(x \cdot(y \cdot z)), \mathrm{f}_{\mathrm{F}}(y)\right\}\right)$.

(5) a fuzzy strongly UP-ideal of $A$ if

(i) $(\forall x \in A) \mathrm{f}_{\mathrm{F}}(0) \geq \mathrm{f}_{\mathrm{F}}(x)$, and

(ii) $(\forall x, y, z \in A)\left(\mathrm{f}_{\mathrm{F}}(x) \geq \min \left\{\mathrm{f}_{\mathrm{F}}((z \cdot y) \cdot(z \cdot x)), \mathrm{f}_{\mathrm{F}}(y)\right\}\right)$.

We know that the notion of fuzzy UP-subalgebras is a generalization of fuzzy near UP-filters, the notion of fuzzy near UP-filters is a generalization of fuzzy UP-filters, the notion of fuzzy UP-filters is a generalization of fuzzy UP-ideals, and the notion of fuzzy UP-ideals is a generalization of fuzzy strongly UP-ideals. Moreover, fuzzy strongly UP-ideals and constant fuzzy sets coincide in UP-algebras.

Satirad and Iampan $[15,16]$ introduced the notion of fuzzy $\mathrm{UP}_{\mathrm{s}}$-subalgebras (resp., fuzzy $\mathrm{UP}_{\mathrm{i}}$-subalgebras, fuzzy near $\mathrm{UP}_{\mathrm{s}}$-filters, fuzzy near $\mathrm{UP}_{\mathrm{i}}$-filters, fuzzy $\mathrm{UP}_{\mathrm{s}}$-filters, fuzzy $\mathrm{UP}_{\mathrm{i}}$-filters, fuzzy $\mathrm{UP}_{\mathrm{s}}$-ideals, fuzzy $\mathrm{UP}_{\mathrm{i}}$ ideals, fuzzy strongly $\mathrm{UP}_{\mathrm{s}}$-ideals, fuzzy strongly $\mathrm{UP}_{\mathrm{i}}$-ideals) of $f$-UP-semigroups as follows:

Definition 1.7. A fuzzy set $\mathrm{F}$ in an $f$-UP-semigroup $A=(A, \cdot, *, 0)$ is called

(1) a fuzzy $U P_{\mathrm{s}}$-subalgebra of $A$ if $\mathrm{F}$ is a fuzzy UP-subalgebra of $(A, \cdot, 0)$ and a fuzzy subsemigroup of $(A, *)$.

(2) a fuzzy $U P_{\mathrm{i}}$-subalgebra of $A$ if $\mathrm{F}$ is a fuzzy UP-subalgebra of $(A, \cdot, 0)$ and a fuzzy ideal of $(A, *)$.

(3) a fuzzy near $U P_{\mathrm{s}}$-filter of $A$ if $\mathrm{F}$ is a fuzzy near $U P$-filter of $(A, \cdot, 0)$ and a fuzzy subsemigroup of $(A, *)$.

(4) a fuzzy near $U P_{\mathrm{i}}$-filter of $A$ if $\mathrm{F}$ is a fuzzy near UP-filter of $(A, \cdot, 0)$ and a fuzzy ideal of $(A, *)$.

(5) a fuzzy $U P_{\mathrm{s}}$-filter of $A$ if $\mathrm{F}$ is a fuzzy $U P$-filter of $(A, \cdot, 0)$ and a fuzzy subsemigroup of $(A, *)$.

(6) a fuzzy $U P_{\mathrm{i}}$-filter of $A$ if $\mathrm{F}$ is a fuzzy UP-filter of $(A, \cdot, 0)$ and a fuzzy ideal of $(A, *)$.

(7) a fuzzy $U P_{\mathrm{s}}$-ideal of $A$ if $\mathrm{F}$ is a fuzzy UP-ideal of $(A, \cdot, 0)$ and a fuzzy subsemigroup of $(A, *)$.

(8) a fuzzy $U P_{\mathrm{i}}$-ideal of $A$ if $\mathrm{F}$ is a fuzzy UP-ideal of $(A, \cdot, 0)$ and a fuzzy ideal of $(A, *)$.

(9) a fuzzy strongly $U P_{\mathrm{s}}$-ideal of $A$ if $\mathrm{F}$ is a fuzzy strongly $U P$-ideal of $(A, \cdot, 0)$ and a fuzzy subsemigroup of $(A, *)$.

(10) a fuzzy strongly $U P_{\mathrm{i}}$-ideal of $A$ if $\mathrm{F}$ is a fuzzy strongly UP-ideal of $(A, \cdot, 0)$ and a fuzzy ideal of $(A, *)$.

Theorem 1.2. [15,16] The intersection of any nonempty family of fuzzy $U P_{\mathrm{s}}$-subalgebras (resp., fuzzy $U P_{\mathrm{i}}$-subalgebras, fuzzy near $U P_{\mathrm{s}}$-filters, fuzzy near $U P_{\mathrm{i}}$-filters, fuzzy $U P_{\mathrm{s}}$-filters, fuzzy $U P_{\mathrm{i}}$-filters, fuzzy $U P_{\mathrm{s}^{-}}$ ideals, fuzzy UP $\mathrm{P}_{\mathrm{i}}$-ideals, fuzzy strongly $U P_{\mathrm{s}}$-ideals, fuzzy strongly $U P_{\mathrm{i}}$-ideals) of an $f$-UP-semigroup is also 
a fuzzy $U P_{\mathrm{s}^{-}}$-subalgebra (resp., fuzzy $U P_{\mathrm{i}}$-subalgebra, fuzzy near $U P_{\mathrm{s}}$-filter, fuzzy near $U P_{\mathrm{i}}$-filter, fuzzy $U P_{\mathrm{s}^{-}}$ filter, fuzzy $U P_{\mathrm{i}}$-filter, fuzzy $U P_{\mathrm{s}}$-ideal, fuzzy $U P_{\mathrm{i}}$-ideal, fuzzy strongly $U P_{\mathrm{s}}$-ideal, fuzzy strongly $U P_{\mathrm{i}}-$ ideal).

Theorem 1.3. [15,16] The union of any nonempty family of fuzzy near UP $P_{\mathrm{i}}$-filters (resp., fuzzy strongly $U P_{\mathrm{s}}$-ideals, fuzzy strongly $U P_{\mathrm{i}}$-ideals) of an $f$-UP-semigroup is also a fuzzy near $U P_{\mathrm{i}}$-filter (resp., fuzzy strongly $U P_{\mathrm{s}}$-ideal, fuzzy strongly $U P_{\mathrm{i}}$-ideal).

\section{Fuzzy Soft Sets over Fully UP-Semigroups}

From now on, we shall let $A$ be an $f$-UP-semigroup $A=(A, \cdot, *, 0)$ and $P$ be a set of parameters. Let $\mathcal{F}(A)$ denotes the set of all fuzzy sets in $A$. A subset $E$ of $P$ is called a set of statistics.

Definition 2.1. Let $E \subseteq P$. A pair $(\widetilde{\mathrm{F}}, E)$ is called a fuzzy soft set over $A$ if $\widetilde{\mathrm{F}}$ is a mapping given by $\widetilde{\mathrm{F}}: E \rightarrow \mathcal{F}(A)$, that is, a fuzzy soft set is a statistic family of fuzzy sets in $A$. In general, for every $e \in E$, $\widetilde{\mathrm{F}}[e]:=\left\{\left(x, \mathrm{f}_{\widetilde{\mathrm{F}}[e]}(x)\right) \mid x \in A\right\}$ is a fuzzy set in $A$ and it is called a fuzzy value set of statistic $e$.

Definition 2.2. Let $\left(\widetilde{\mathrm{F}}, E_{1}\right)$ and $\left(\widetilde{\mathrm{G}}, E_{2}\right)$ be two fuzzy soft sets over a common universe $U$. The union [10] of $\left(\widetilde{\mathrm{F}}, E_{1}\right)$ and $\left(\widetilde{\mathrm{G}}, E_{2}\right)$ is defined to be the fuzzy soft set $\left(\widetilde{\mathrm{F}}, E_{1}\right) \cup\left(\widetilde{\mathrm{G}}, E_{2}\right)=(\widetilde{\mathrm{H}}, E)$ satisfying the following conditions:

(i) $E=E_{1} \cup E_{2}$ and

(ii) for all $e \in E$,

$$
\widetilde{\mathrm{H}}[e]= \begin{cases}\widetilde{\mathrm{F}}[e] & \text { if } e \in E_{1} \backslash E_{2} \\ \widetilde{\mathrm{G}}[e] & \text { if } e \in E_{2} \backslash E_{1} \\ \widetilde{\mathrm{F}}[e] \cup \widetilde{\mathrm{G}}[e] & \text { if } e \in E_{1} \cap E_{2} .\end{cases}
$$

The restricted union [13] of $\left(\widetilde{\mathrm{F}}, E_{1}\right)$ and $\left(\widetilde{\mathrm{G}}, E_{2}\right)$ is defined to be the fuzzy soft set $\left(\widetilde{\mathrm{F}}, E_{1}\right) \cup\left(\widetilde{\mathrm{G}}, E_{2}\right)=(\widetilde{\mathrm{H}}, E)$ satisfying the following conditions:

(i) $E=E_{1} \cap E_{2} \neq \emptyset$ and

(ii) $\widetilde{\mathrm{H}}[e]=\widetilde{\mathrm{F}}[e] \cup \widetilde{\mathrm{G}}[e]$ for all $e \in E$.

Definition 2.3. [10] Let $\left(\widetilde{\mathrm{F}}, E_{1}\right)$ and $\left(\widetilde{\mathrm{G}}, E_{2}\right)$ be two fuzzy soft sets over a common universe $U$. The OR of $\left(\widetilde{\mathrm{F}}, E_{1}\right)$ and $\left(\widetilde{\mathrm{G}}, E_{2}\right)$ is defined to be the fuzzy soft set $\left(\widetilde{\mathrm{F}}, E_{1}\right) \vee\left(\widetilde{\mathrm{G}}, E_{2}\right)=(\widetilde{\mathrm{H}}, E)$ satisfying the following conditions:

(i) $E=E_{1} \times E_{2}$ and

(ii) $\widetilde{\mathrm{H}}\left[e_{1}, e_{2}\right]=\widetilde{\mathrm{F}}\left[e_{1}\right] \cup \widetilde{\mathrm{G}}\left[e_{2}\right]$ for all $\left(e_{1}, e_{2}\right) \in E$.

Definition 2.4. Let $\left(\widetilde{\mathrm{F}}, E_{1}\right)$ and $\left(\widetilde{\mathrm{G}}, E_{2}\right)$ be two fuzzy soft sets over a common universe $U$. The extended intersection [13] of $\left(\widetilde{\mathrm{F}}, E_{1}\right)$ and $\left(\widetilde{\mathrm{G}}, E_{2}\right)$ is defined to be the fuzzy soft set $\left(\widetilde{\mathrm{F}}, E_{1}\right) \cap\left(\widetilde{\mathrm{G}}, E_{2}\right)=(\widetilde{\mathrm{H}}, E)$ satisfying the following conditions: 
(i) $E=E_{1} \cup E_{2}$ and

(ii) for all $e \in E$,

$$
\widetilde{\mathrm{H}}[e]= \begin{cases}\widetilde{\mathrm{F}}[e] & \text { if } e \in E_{1} \backslash E_{2} \\ \widetilde{\mathrm{G}}[e] & \text { if } e \in E_{2} \backslash E_{1} \\ \widetilde{\mathrm{F}}[e] \cap \widetilde{\mathrm{G}}[e] & \text { if } e \in E_{1} \cap E_{2} .\end{cases}
$$

The intersection [1] of $\left(\widetilde{\mathrm{F}}, E_{1}\right)$ and $\left(\widetilde{\mathrm{G}}, E_{2}\right)$ is defined to be the fuzzy soft set $\left(\widetilde{\mathrm{F}}, E_{1}\right)$ ก $\left(\widetilde{\mathrm{G}}, E_{2}\right)=(\widetilde{\mathrm{H}}, E)$ satisfying the following conditions:

(i) $E=E_{1} \cap E_{2} \neq \emptyset$ and

(ii) $\widetilde{\mathrm{H}}[e]=\widetilde{\mathrm{F}}[e] \cap \widetilde{\mathrm{G}}[e]$ for all $e \in E$.

Definition 2.5. [10] Let $\left(\widetilde{\mathrm{F}}, E_{1}\right)$ and $\left(\widetilde{\mathrm{G}}, E_{2}\right)$ be two fuzzy soft sets over a common universe $U$. The AND of $\left(\widetilde{\mathrm{F}}, E_{1}\right)$ and $\left(\widetilde{\mathrm{G}}, E_{2}\right)$ is defined to be the fuzzy soft set $\left(\widetilde{\mathrm{F}}, E_{1}\right) \wedge\left(\widetilde{\mathrm{G}}, E_{2}\right)=(\widetilde{\mathrm{H}}, E)$ satisfying the following conditions:

(i) $E=E_{1} \times E_{2}$ and

(ii) $\widetilde{\mathrm{H}}\left[e_{1}, e_{2}\right]=\widetilde{\mathrm{F}}\left[e_{1}\right] \cap \widetilde{\mathrm{G}}\left[e_{2}\right]$ for all $\left(e_{1}, e_{2}\right) \in E$.

Definition 2.6. A fuzzy soft set $(\widetilde{\mathrm{F}}, E)$ over $A$ is called a fuzzy soft $U P_{\mathrm{s}}$-subalgebra based on $e \in E$ (we shortly call an e-fuzzy soft $U P_{\mathrm{s}}$-subalgebra) of $A$ if a fuzzy set $\widetilde{\mathrm{F}}[e]$ in $A$ is a fuzzy $U P_{\mathrm{s}}$-subalgebra of $A$. If $(\widetilde{\mathrm{F}}, E)$ is an e-fuzzy soft $U P_{\mathrm{s}}$-subalgebra of $A$ for all $e \in E$, we say that $(\widetilde{\mathrm{F}}, E)$ is a fuzzy soft $U P_{\mathrm{s}}$-subalgebra of $A$.

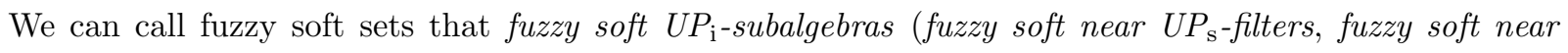
$U P_{\mathrm{i}}$-filters, fuzzy soft $U P_{\mathrm{s}}$-filters, fuzzy soft $U P_{\mathrm{i}}$-filters, fuzzy soft $U P_{\mathrm{s}}$-ideals, fuzzy soft $U P_{\mathrm{i}}$-ideals, fuzzy soft strongly $U P_{\mathrm{s}}$-ideals, and fuzzy soft strongly $U P_{\mathrm{i}}$-ideals) based on a statistic or fuzzy soft $U P_{\mathrm{i}}$-subalgebras (fuzzy soft near $U P_{\mathrm{s}}$-filters, fuzzy soft near $U P_{\mathrm{i}}$-filters, fuzzy soft $U P_{\mathrm{s}}$-filters, fuzzy soft $U P_{\mathrm{i}}$-filters, fuzzy soft $U P_{\mathrm{s}}$-ideals, fuzzy soft $U P_{\mathrm{i}}$-ideals, fuzzy soft strongly $U P_{\mathrm{s}}$-ideals, and fuzzy soft strongly $\left.U P_{\mathrm{i}}-i d e a l s\right)$ of $A$ if fuzzy soft sets satisfy statement in Definition 2.6.

We will introduce the notions of the restricted union, the union, the intersection, the extended intersection, the AND, and the OR of any fuzzy soft sets and apply to $f$-UP-semigroups.

Definition 2.7. Let $\left\{\left(\widetilde{\mathrm{F}}_{i}, E_{i}\right) \mid i \in I\right\}$ be a nonempty family of fuzzy soft sets over a common universe $U$ where $I$ is an arbitrary index set. The restricted union of $\left(\widetilde{\mathrm{F}}_{i}, E_{i}\right)$ is defined to be the fuzzy soft set $\bigcup_{i \in I}\left(\widetilde{\mathrm{F}}_{i}, E_{i}\right)=(\widetilde{\mathrm{F}}, E)$ satisfying the following conditions:

(i) $E=\bigcap_{i \in I} E_{i} \neq \emptyset$ and

(ii) $\widetilde{\mathrm{F}}[e]=\bigcup_{i \in I} \widetilde{\mathrm{F}}_{i}[e]$ for all $e \in E$. 
Theorem 2.1. The restricted union of family of fuzzy soft near UP-filters of $A$ is also a fuzzy soft near $U P_{\mathrm{i}}-$ filter.

Proof. Let $\left(\widetilde{\mathrm{F}}_{i}, E_{i}\right)$ be a fuzzy soft near $\mathrm{UP}_{\mathrm{i}}$-filters of $A$ for all $i \in I$. Assume that $\bigcup_{i \in I}\left(\widetilde{\mathrm{F}}_{i}, E_{i}\right)=(\widetilde{\mathrm{F}}, E)$ be the restricted union of $\left(\widetilde{\mathrm{F}}_{i}, E_{i}\right)$ for all $i \in I$. Then $E=\bigcap_{i \in I} E_{i} \neq \emptyset$. Let $e \in E$. By Theorem 1.3, we have $\widetilde{\mathrm{F}}[e]=\bigcup_{i \in I} \widetilde{\mathrm{F}}_{i}[e]$ is a fuzzy near $\mathrm{UP}_{\mathrm{i}}$-filter of $A$. Therefore, $(\widetilde{\mathrm{F}}, E)$ is an $e$-fuzzy soft near $\mathrm{UP}_{\mathrm{i}}$-filter of $A$. But since $e$ is an arbitrary statistic of $E$, we have $(\widetilde{\mathrm{F}}, E)$ is a fuzzy soft near $\mathrm{UP}_{\mathrm{i}}$-filter of $A$.

In the same way as Theorem 2.1, we can use Theorem 1.3 to prove that the restricted union of family of fuzzy soft strongly $\mathrm{UP}_{\mathrm{s}}$-ideals (resp., fuzzy soft strongly $\mathrm{UP}_{\mathrm{i}}$-ideals) of $A$ is also a fuzzy soft strongly $\mathrm{UP}_{\mathrm{s}}$-ideal (resp., fuzzy soft strongly $\mathrm{UP}_{\mathrm{i}}$-ideal).

Definition 2.8. Let $\left\{\left(\widetilde{\mathrm{F}}_{i}, E_{i}\right) \mid i \in I\right\}$ be a nonempty family of fuzzy soft sets over a common universe $U$ where $I$ is an arbitrary index set. The union of $\left(\widetilde{\mathrm{F}}_{i}, E_{i}\right)$ is defined to be the fuzzy soft set $\bigcup_{i \in I}\left(\widetilde{\mathrm{F}}_{i}, E_{i}\right)=(\widetilde{\mathrm{F}}, E)$ satisfying the following conditions:

(i) $E=\bigcup_{i \in I} E_{i}$ and

(ii) $\widetilde{\mathrm{F}}[e]=\bigcup_{j \in J} \widetilde{\mathrm{F}}_{j}[e]$ for all $e \in E$ with $e \in \bigcap_{j \in J} E_{j}-\bigcup_{k \in I-J} E_{k}$ where $\emptyset \neq J \subseteq I$.

Theorem 2.2. The union of family of fuzzy soft near UP $\mathrm{i}$-filters of $A$ is also a fuzzy soft near $U P_{\mathrm{i}}$-filter.

Proof. Let $\left(\widetilde{\mathrm{F}}_{i}, E_{i}\right)$ be a fuzzy soft near $\mathrm{UP}_{\mathrm{i}}$-filters of $A$ for all $i \in I$. Assume that $\bigcap_{i \in I}\left(\widetilde{\mathrm{F}}_{i}, E_{i}\right)=(\widetilde{\mathrm{F}}, E)$ be the union of $\left(\widetilde{\mathrm{F}}_{i}, E_{i}\right)$ for all $i \in I$. Then $E=\bigcup_{i \in I} E_{i}$. Let $e \in E$.

Case 1: $|J|=|I|$. By Theorem 2.1, we have $\widetilde{\mathrm{F}}[e]=\bigcap_{i \in I} \widetilde{\mathrm{F}}_{i}[e]$ is a fuzzy near $\mathrm{UP}_{\mathrm{i}}$-filter of $A$.

Case 2: $|J|=1$, that is, $J$ is a singleton set. Then $\widetilde{\mathrm{F}}[e]=\bigcap_{j \in\{j\}} \widetilde{\mathrm{F}}_{j}[e]=\widetilde{\mathrm{F}}_{j}[e]$ is a fuzzy near UP -filter of $A$.

Case 3: $1<|J|<|I|$. Then $\widetilde{\mathrm{F}}[e]=\bigcap_{j \in J} \widetilde{\mathrm{F}}_{j}[e]$. Since $e \in E_{j}$ for all $j \in J$ and $e \notin E_{k}$ for some $k \in I-J$ and by same Case 1 , we have $\widetilde{\mathrm{F}}[e]$ is a fuzzy near $\mathrm{UP}_{\mathrm{i}}$-filter of $A$.

Therefore, $(\widetilde{\mathrm{F}}, E)$ is an $e$-fuzzy soft near $\mathrm{UP}_{\mathrm{i}}$-filter of $A$. But since $e$ is an arbitrary statistic of $E$, we have $(\widetilde{\mathrm{F}}, E)$ is a fuzzy soft near $\mathrm{UP}_{\mathrm{i}}$-filter of $A$.

In the same way as Theorem 2.2 , we can prove that the union of family of fuzzy soft strongly $\mathrm{UP}_{\mathrm{s}}$-ideals (resp., fuzzy soft strongly $\mathrm{UP}_{\mathrm{i}}$-ideals) of $A$ is also a fuzzy soft strongly $\mathrm{UP}_{\mathrm{s}}$-ideal (resp., fuzzy soft strongly $\mathrm{UP}_{\mathrm{i}}$-ideal).

In [16], we show that the union of two fuzzy soft $\mathrm{UP}_{\mathrm{s}}$-subalgebras (resp., fuzzy soft $\mathrm{UP}_{\mathrm{i}}$-subalgebras, fuzzy soft near $\mathrm{UP}_{\mathrm{s}}$-filters, fuzzy soft $\mathrm{UP}_{\mathrm{s}}$-filters, fuzzy soft $\mathrm{UP}_{\mathrm{i}}$-filters, fuzzy soft $\mathrm{UP}_{\mathrm{s}}$-ideals, fuzzy soft $\mathrm{UP}_{\mathrm{i}}$-ideals) of $A$ is not fuzzy soft $\mathrm{UP}_{\mathrm{s}}$-subalgebra (resp., fuzzy soft $\mathrm{UP}_{\mathrm{i}}$-subalgebra, fuzzy soft near $\mathrm{UP}_{\mathrm{s}}$-filter, fuzzy soft $\mathrm{UP}_{\mathrm{s}}$-filter, fuzzy soft $\mathrm{UP}_{\mathrm{i}}$-filter, fuzzy soft $\mathrm{UP}_{\mathrm{s}}$-ideal, fuzzy soft $\mathrm{UP}_{\mathrm{i}}$-ideal). 
Definition 2.9. Let $\left\{\left(\widetilde{\mathrm{F}}_{i}, E_{i}\right) \mid i \in I\right\}$ be a nonempty family of fuzzy soft sets over a common universe $U$ where $I$ is an arbitrary index set. The intersection of $\left(\widetilde{\mathrm{F}}_{i}, E_{i}\right)$ is defined to be the fuzzy soft set $\bigcap_{i \in I}\left(\widetilde{\mathrm{F}}_{i}, E_{i}\right)=$ $(\widetilde{\mathrm{F}}, E)$ satisfying the following conditions:

(i) $E=\bigcap_{i \in I} E_{i} \neq \emptyset$ and

(ii) $\widetilde{\mathrm{F}}[e]=\bigcap_{i \in I} \widetilde{\mathrm{F}}_{i}[e]$ for all $e \in E$.

Theorem 2.3. The intersection of family of fuzzy soft $U P_{\mathrm{s}^{-}}$subalgebras of $A$ is also a fuzzy soft $U P_{\mathrm{s}^{-}}$ subalgebra.

Proof. Let $\left(\widetilde{\mathrm{F}}_{i}, E_{i}\right)$ be a fuzzy soft $\mathrm{UP}_{\mathrm{s}}$-subalgebras of $A$ for all $i \in I$. Assume that $\bigcap_{i \in I}\left(\widetilde{\mathrm{F}}_{i}, E_{i}\right)=(\widetilde{\mathrm{F}}, E)$ is the intersection of $\left(\widetilde{\mathrm{F}}_{i}, E_{i}\right)$ for all $i \in I$. Then $E=\bigcap_{i \in I} E_{i} \neq \emptyset$. Let $e \in E$. By Theorem 1.2, we have $\widetilde{\mathrm{F}}[e]=\bigcap_{i \in I} \widetilde{\mathrm{F}}_{i}[e]$ is a fuzzy $\mathrm{UP}_{\mathrm{s}}$-subalgebra of $A$. Therefore, $(\widetilde{\mathrm{F}}, E)$ is an $e$-fuzzy soft $\mathrm{UP}_{\mathrm{s}}$-subalgebra of $A$. But since $e$ is an arbitrary statistic of $E$, we have $(\widetilde{\mathrm{F}}, E)$ is a fuzzy soft $\mathrm{UP}_{\mathrm{s}}$-subalgebra of $A$.

In the same way as Theorem 2.3, we can use Theorem 1.2 to prove that the intersection of family of fuzzy soft $\mathrm{UP}_{\mathrm{i}}$-subalgebras (resp., fuzzy soft near $\mathrm{UP}_{\mathrm{s}}$-filters, fuzzy soft near $\mathrm{UP}_{\mathrm{i}}$-filters, fuzzy soft $\mathrm{UP}_{\mathrm{s}}$-filters, fuzzy soft $\mathrm{UP}_{\mathrm{i}}$-filters, fuzzy soft $\mathrm{UP}_{\mathrm{s}}$-ideals, fuzzy soft $\mathrm{UP}_{\mathrm{i}}$-ideals, fuzzy soft strongly $\mathrm{UP}_{\mathrm{s}}$-ideals, fuzzy soft strongly $\mathrm{UP}_{\mathrm{i}}$-ideals) of $A$ is also a fuzzy soft $\mathrm{UP}_{\mathrm{i}}$-subalgebra (resp., fuzzy soft near $\mathrm{UP}_{\mathrm{s}}$-filter, fuzzy soft near $\mathrm{UP}_{\mathrm{i}}$-filter, fuzzy soft $\mathrm{UP}_{\mathrm{s}}$-filter, fuzzy soft $\mathrm{UP}_{\mathrm{i}}$-filter, fuzzy soft $\mathrm{UP}_{\mathrm{s}}$-ideal, fuzzy soft $\mathrm{UP}_{\mathrm{i}}$-ideal, fuzzy soft strongly $\mathrm{UP}_{\mathrm{s}}$-ideal, fuzzy soft strongly $\mathrm{UP}_{\mathrm{i}}$-ideal).

Definition 2.10. Let $\left\{\left(\widetilde{\mathrm{F}}_{i}, E_{i}\right) \mid i \in I\right\}$ be a nonempty family of fuzzy soft sets over a common universe $U$ where $I$ is an arbitrary index set. The extended intersection of $\left(\widetilde{\mathrm{F}}_{i}, E_{i}\right)$ is defined to be the fuzzy soft set $\bigcap_{i \in I}\left(\widetilde{\mathrm{F}}_{i}, E_{i}\right)=(\widetilde{\mathrm{F}}, E)$ satisfying the following conditions:

(i) $E=\bigcup_{i \in I} E_{i}$ and

(ii) $\widetilde{\mathrm{F}}[e]=\bigcap_{j \in J} \widetilde{\mathrm{F}}_{j}[e]$ for all $e \in E$ with $e \in \bigcap_{j \in J} E_{j}-\bigcup_{k \in I-J} E_{k}$ where $\emptyset \neq J \subseteq I$.

Theorem 2.4. The extended intersection of family of fuzzy soft $U P_{\mathrm{s}}$-subalgebras of $A$ is also a fuzzy soft $U P_{\mathrm{s}}$-subalgebra.

Proof. Let $\left(\widetilde{\mathrm{F}}_{i}, E_{i}\right)$ be a fuzzy soft $\mathrm{UP}_{\mathrm{s}}$-subalgebras of $A$ for all $i \in I$. Assume that $\bigcap_{i \in I}\left(\widetilde{\mathrm{F}}_{i}, E_{i}\right)=(\widetilde{\mathrm{F}}, E)$ is the extended intersection of $\left(\widetilde{\mathrm{F}}_{i}, E_{i}\right)$ for all $i \in I$. Then $E=\bigcup_{i \in I} E_{i}$. Let $e \in E$.

Case 1: $|J|=|I|$. By Theorem 2.3, we have $\widetilde{\mathrm{F}}[e]=\bigcap_{i \in I} \widetilde{\mathrm{F}}_{i}[e]$ is a fuzzy UP $\mathrm{UP}_{\mathrm{s}}$-subalgebra of $A$.

Case $2:|J|=1$, that is, $J$ is a singleton set. Then $\widetilde{\mathrm{F}}[e]=\bigcap_{j \in\{j\}} \widetilde{\mathrm{F}}_{j}[e]=\widetilde{\mathrm{F}}_{j}[e]$ is a fuzzy $\mathrm{UP}_{\mathrm{s}}$-subalgebra of $A$.

Case 3: $1<|J|<|I|$. Then $\widetilde{\mathrm{F}}[e]=\bigcap_{j \in J} \widetilde{\mathrm{F}}_{j}[e]$. Since $e \in E_{j}$ for all $j \in J$ and $e \notin E_{k}$ for some $k \in I-J$ and by same Case 1 , we have $\widetilde{\mathrm{F}}[e]$ is a fuzzy $\mathrm{UP}_{\mathrm{s}}$-subalgebra of $A$. 
Therefore, $(\widetilde{\mathrm{F}}, E)$ is an $e$-fuzzy soft $\mathrm{UP}_{\mathrm{s}}$-subalgebra of $A$. But since $e$ is an arbitrary statistic of $E$, we have $(\widetilde{\mathrm{F}}, E)$ is a fuzzy soft $\mathrm{UP}_{\mathrm{s}}$-subalgebra of $A$.

In the same way as Theorem 2.4, we can prove that the extended intersection of family of fuzzy soft UP $\mathrm{i}^{-}$ subalgebras (resp., fuzzy soft near $\mathrm{UP}_{\mathrm{s}}$-filters, fuzzy soft near $\mathrm{UP}_{\mathrm{i}}$-filters, fuzzy soft $\mathrm{UP}_{\mathrm{s}}$-filters, fuzzy soft $\mathrm{UP}_{\mathrm{i}}$-filters, fuzzy soft $\mathrm{UP}_{\mathrm{s}}$-ideals, fuzzy soft $\mathrm{UP}_{\mathrm{i}}$-ideals, fuzzy soft strongly $\mathrm{UP}_{\mathrm{s}}$-ideals, fuzzy soft strongly

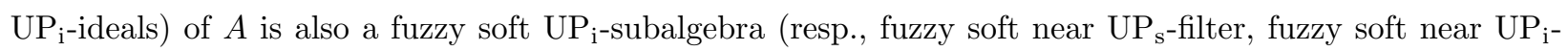
filter, fuzzy soft $\mathrm{UP}_{\mathrm{s}}$-filter, fuzzy soft $\mathrm{UP}_{\mathrm{i}}$-filter, fuzzy soft $\mathrm{UP}_{\mathrm{s}}$-ideal, fuzzy soft $\mathrm{UP}_{\mathrm{i}}$-ideal, fuzzy soft strongly $\mathrm{UP}_{\mathrm{s}}$-ideal, fuzzy soft strongly $\mathrm{UP}_{\mathrm{i}}$-ideal).

Definition 2.11. Let $\left\{\left(\widetilde{\mathrm{F}}_{i}, E_{i}\right) \mid i \in I\right\}$ be a nonempty family of fuzzy soft sets over a common universe $U$ where $I$ is an arbitrary index set. The $\mathrm{AND}$ of $\left(\widetilde{\mathrm{F}}_{i}, E_{i}\right)$ is defined to be the fuzzy soft set $\bigwedge_{i \in I}\left(\widetilde{\mathrm{F}}_{i}, E_{i}\right)=(\widetilde{\mathrm{F}}, E)$ satisfying the following conditions:

(i) $E=\prod_{i \in I} E_{i}$ and

(ii) $\widetilde{\mathrm{F}}\left[\left(e_{i}\right)_{i \in I}\right]=\bigcap_{i \in I} \widetilde{\mathrm{F}}_{i}\left[e_{i}\right]$ for all $\left(e_{i}\right)_{i \in I} \in E$.

Theorem 2.5. The AND of family of fuzzy soft UP $P_{\mathrm{s}}$-subalgebras of $A$ is also a fuzzy soft UP $P_{\mathrm{s}}-$ subalgebra.

Proof. Let $\left(\widetilde{\mathrm{F}}_{i}, E_{i}\right)$ be a fuzzy soft $\mathrm{UP}_{\mathrm{s}}$-subalgebras of $A$ for all $i \in I$. By means of Definition 2.11 , we assume that $\bigwedge_{i \in I}\left(\widetilde{\mathrm{F}}_{i}, E_{i}\right)=(\widetilde{\mathrm{F}}, E)$ such that $E=\prod_{i \in I} E_{i}$ and $\widetilde{\mathrm{F}}\left[\left(e_{i}\right)_{i \in I}\right]=\bigcap_{i \in I} \widetilde{\mathrm{F}}_{i}\left[e_{i}\right]$ for all $\left(e_{i}\right)_{i \in I} \in E$. Assume that $e=\left(e_{i}\right)_{i \in I} \in E$ and let $x, y \in A$. Then

$$
\begin{aligned}
\mathrm{f}_{\widetilde{\mathrm{F}}[e]}(x \cdot y) & =\mathrm{f}_{\bigcap_{i \in I} \widetilde{\mathrm{F}}_{i}\left[e_{i}\right]}(x \cdot y) \\
& =\inf \left\{\mathrm{f}_{\widetilde{\mathrm{F}}_{i}\left[e_{i}\right]}(x \cdot y)\right\}_{i \in I} \\
& \geq \inf \left\{\min \left\{\mathrm{f}_{\widetilde{\mathrm{F}}_{i}\left[e_{i}\right]}(x), \mathrm{f}_{\widetilde{\mathrm{F}}_{i}\left[e_{i}\right]}(y)\right\}\right\}_{i \in I} \\
& =\min \left\{\inf \left\{\mathrm{f}_{\widetilde{\mathrm{F}}_{i}\left[e_{i}\right]}(x)\right\}_{i \in I}, \inf \left\{\mathrm{f}_{\widetilde{\mathrm{F}}_{i}\left[e_{i}\right]}(y)\right\}_{i \in I}\right\} \\
& =\min \left\{\mathrm{f}_{\bigcap_{i \in I}} \widetilde{\mathrm{F}}_{i}\left[e_{i}\right]\right. \\
& =\min \left\{\mathrm{f}_{\left.\widetilde{\mathrm{F}}_{[e]}\right]}(x), \mathrm{f}_{\left.\widetilde{\mathrm{F}}_{[e]}\right]}(y)\right\}, \text { and } \\
\mathrm{f}_{\widetilde{\mathrm{F}}[e]}(x * y) & \left.=\mathrm{f}_{\bigcap_{i \in I}\left[\widetilde{\mathrm{F}}_{i}\right]\left[e_{i}\right]}(x * y)\right\} \\
& =\inf \left\{\mathrm{f}_{\widetilde{\mathrm{F}}_{i}\left[e_{i}\right]}(x * y)\right\}_{i \in I} \\
& \geq \inf \left\{\min \left\{\mathrm{f}_{\widetilde{\mathrm{F}}_{i}\left[e_{i}\right]}(x), \mathrm{f}_{\widetilde{\mathrm{F}}_{i}\left[e_{i}\right]}(y)\right\}\right\}_{i \in I} \\
& \left.\left.=\min \left\{\inf \left\{\mathrm{f}_{\widetilde{\mathrm{F}}_{i}\left[e_{i}\right]}(x)\right\}\right\}_{i \in I}, \inf \left\{\mathrm{f}_{\widetilde{\mathrm{F}}_{i}\left[e_{i}\right]}(y)\right\}\right]_{i \in I}\right\} \\
& =\min \left\{\mathrm{f}_{\bigcap_{i \in I} \widetilde{\mathrm{F}}_{i}\left[e_{i}\right]}(x), \mathrm{f}_{\bigcap_{i \in I} \widetilde{\mathrm{F}}_{i}\left[e_{i}\right]}(y)\right\} \\
& =\min \left\{\mathrm{f}_{\widetilde{\mathrm{F}}_{[}[e]}(x), \mathrm{f}_{\widetilde{\mathrm{F}}[e]}(y)\right\} .
\end{aligned}
$$


Therefore, $\widetilde{\mathrm{F}}[e]$ is a fuzzy $\mathrm{UP}_{\mathrm{s}}$-subalgebra of $A$, that is, $(\widetilde{\mathrm{F}}, E)$ is an $e$-fuzzy soft $\mathrm{UP}_{\mathrm{s}}$-subalgebra of $A$. But since $e$ is an arbitrary statistic of $E$, we have $(\widetilde{\mathrm{F}}, E)$ is a fuzzy soft $\mathrm{UP}_{\mathrm{s}}$-subalgebra of $A$.

In the same way as Theorem 2.5, we can use Theorem 1.2 to prove that the AND of family of fuzzy soft $\mathrm{UP}_{\mathrm{i}}$-subalgebras (resp., fuzzy soft near $\mathrm{UP}_{\mathrm{s}}$-filters, fuzzy soft near $\mathrm{UP}_{\mathrm{i}}$-filters, fuzzy soft $\mathrm{UP}_{\mathrm{s}}$-filters, fuzzy soft $\mathrm{UP}_{\mathrm{i}}$-filters, fuzzy soft $\mathrm{UP}_{\mathrm{s}}$-ideals, fuzzy soft $\mathrm{UP}_{\mathrm{i}}$-ideals, fuzzy soft strongly $\mathrm{UP}_{\mathrm{s}}$-ideals, fuzzy soft strongly $\mathrm{UP}_{\mathrm{i}}$-ideals) of $A$ is also a fuzzy soft $\mathrm{UP}_{\mathrm{i}}$-subalgebra (resp., fuzzy soft near $\mathrm{UP}_{\mathrm{s}}$-filter, fuzzy soft near $\mathrm{UP}_{\mathrm{i}}$-filter, fuzzy soft $\mathrm{UP}_{\mathrm{s}}$-filter, fuzzy soft $\mathrm{UP}_{\mathrm{i}}$-filter, fuzzy soft $\mathrm{UP}_{\mathrm{s}}$-ideal, fuzzy soft $\mathrm{UP}_{\mathrm{i}}$-ideal, fuzzy soft strongly $\mathrm{UP}_{\mathrm{s}}$-ideal, fuzzy soft strongly $\mathrm{UP}_{\mathrm{i}}$-ideal).

Definition 2.12. Let $\left\{\left(\widetilde{\mathrm{F}}_{i}, E_{i}\right) \mid i \in I\right\}$ be a nonempty family of fuzzy soft sets over a common universe $U$ where $I$ is an arbitrary index set. The OR of $\left(\widetilde{\mathrm{F}}_{i}, E_{i}\right)$ is defined to be the fuzzy soft set $\bigvee_{i \in I}\left(\widetilde{\mathrm{F}}_{i}, E_{i}\right)=(\widetilde{\mathrm{F}}, E)$ satisfying the following conditions:

(i) $E=\prod_{i \in I} E_{i}$ and

(ii) $\widetilde{\mathrm{F}}\left[\left(e_{i}\right)_{i \in I}\right]=\bigcup_{i \in I} \widetilde{\mathrm{F}}_{i}\left[e_{i}\right]$ for all $\left(e_{i}\right)_{i \in I} \in E$.

Theorem 2.6. The OR of family of fuzzy soft near $U P_{\mathrm{i}}$-filters of $A$ is also a fuzzy soft near $U P_{\mathrm{i}}$-filter.

Proof. Let $\left(\widetilde{\mathrm{F}}_{i}, E_{i}\right)$ be a fuzzy soft near $\mathrm{UP}_{\mathrm{i}}$-filters of $A$ for all $i \in I$. By means of Definition 2.12, we assume that $\bigvee_{i \in I}\left(\widetilde{\mathrm{F}}_{i}, E_{i}\right)=(\widetilde{\mathrm{F}}, E)$ such that $E=\prod_{i \in I} E_{i}$ and $\widetilde{\mathrm{F}}\left[\left(e_{i}\right)_{i \in I}\right]=\bigcup_{i \in I} \widetilde{\mathrm{F}}_{i}\left[e_{i}\right]$ for all $\left(e_{i}\right)_{i \in I} \in E$. Assume that $e=\left(e_{i}\right)_{i \in I} \in E$ and let $x, y \in A$. Then

$$
\begin{aligned}
\mathrm{f}_{\widetilde{\mathrm{F}}[e]}(0) & =\mathrm{f}_{\cup_{i \in I} \widetilde{\mathrm{F}}_{i}\left[e_{i}\right]}(0) \\
& =\sup \left\{\mathrm{f}_{\widetilde{\mathrm{F}}_{i}\left[e_{i}\right]}(0)\right\}_{i \in I} \\
& \geq \sup \left\{\mathrm{f}_{\widetilde{\mathrm{F}}_{i}\left[e_{i}\right]}(x)\right\}_{i \in I} \\
& =\mathrm{f}_{\cup_{i \in I}} \widetilde{\mathrm{F}}_{i}\left[e_{i}\right] \\
& =\mathrm{f}_{\widetilde{\mathrm{F}}[e]}(x), \\
\mathrm{f}_{\widetilde{\mathrm{F}}[e]}(x \cdot y) & =\mathrm{f}_{\bigcup_{i \in I}} \widetilde{\mathrm{F}}_{i}\left[e_{i}\right] \\
& =\sup \left\{\mathrm{f}_{\widetilde{\mathrm{F}}_{i}\left[e_{i}\right]}(x \cdot y)\right\}_{i \in I} \\
& \geq \sup \left\{\mathrm{f}_{\widetilde{\mathrm{F}}_{i}\left[e_{i}\right]}(y)\right\}_{i \in I} \\
& =\mathrm{f}_{\bigcup_{i \in I}} \widetilde{\mathrm{F}}_{i}\left[e_{i}\right] \\
& =\mathrm{f}_{\widetilde{\mathrm{F}}[e]}(y), \text { and }
\end{aligned}
$$




$$
\begin{aligned}
\mathrm{f}_{\widetilde{\mathrm{F}}[e]}(x * y) & =\mathrm{f}_{\cup_{i \in I} \widetilde{\mathrm{F}}_{i}\left[e_{i}\right]}(x * y) \\
& =\sup \left\{\mathrm{f}_{\widetilde{\mathrm{F}}_{i}\left[e_{i}\right]}(x * y)\right\}_{i \in I} \\
& \geq \sup \left\{\max \left\{\mathrm{f}_{\widetilde{\mathrm{F}}_{i}\left[e_{i}\right]}(x), \mathrm{f}_{\widetilde{\mathrm{F}}_{i}\left[e_{i}\right]}(y)\right\}\right\}_{i \in I} \\
& =\max \left\{\sup \left\{\mathrm{f}_{\widetilde{\mathrm{F}}_{i}\left[e_{i}\right]}(x)\right\}_{i \in I}, \sup \left\{\mathrm{f}_{\widetilde{\mathrm{F}}_{i}\left[e_{i}\right]}(y)\right\}_{i \in I}\right\} \\
& =\max \left\{\mathrm{f}_{\bigcap_{i \in I} \widetilde{\mathrm{F}}_{i}\left[e_{i}\right]}(x), \mathrm{f}_{\bigcap_{i \in I} \widetilde{\mathrm{F}}_{i}\left[e_{i}\right]}(y)\right\} \\
& =\max \left\{\mathrm{f}_{\widetilde{\mathrm{F}}[e]}(x), \mathrm{f}_{\widetilde{\mathrm{F}}[e]}(y)\right\} .
\end{aligned}
$$

Therefore, $\widetilde{\mathrm{F}}[e]$ is a fuzzy near $\mathrm{UP}_{\mathrm{i}}$-filter of $A$, that is, $(\widetilde{\mathrm{F}}, E)$ is an $e$-fuzzy soft near $\mathrm{UP}_{\mathrm{i}}$-filter of $A$. But since $e$ is an arbitrary statistic of $E$, we have $(\widetilde{\mathrm{F}}, E)$ is a fuzzy soft near $\mathrm{UP}_{\mathrm{i}}$-filter of $A$.

In the same way as Theorem 2.6, we can use Theorem 1.3 to prove that the OR of family of fuzzy soft strongly $\mathrm{UP}_{\mathrm{s}}$-ideals (resp., fuzzy soft strongly $\mathrm{UP}_{\mathrm{i}}$-ideals) of $A$ is also a fuzzy soft strongly $\mathrm{UP}_{\mathrm{s}}$-ideal (resp., fuzzy soft strongly $\mathrm{UP}_{\mathrm{i}}$-ideal).

\begin{tabular}{|c|c|c|c|c|c|c|c|c|c|}
\hline & $X$ & 7 & 6 & 5 & $*$ & $X$ & 7 & 6 & 5 \\
\hline$X$ & $X$ & 7 & 6 & 5 & $X$ & $X$ & $X$ & $X$ & $X$ \\
\hline 7 & $X$ & $X$ & 6 & 5 & 7 & $X$ & $X$ & $X$ & $X$ \\
\hline 6 & $X$ & 7 & $X$ & 5 & 6 & $X$ & $X$ & $X$ & 7 \\
\hline 5 & $X$ & 7 & 6 & $X$ & 5 & $X$ & $X$ & 7 & $X$ \\
\hline
\end{tabular}

The following example shows that the OR of two fuzzy soft $\mathrm{UP}_{\mathrm{s}^{-}}$subalgebras of $A$ is not fuzzy soft $\mathrm{UP}_{\mathrm{s}^{-}}$ subalgebra.

Example 2.1. Let $A$ be the set of four series of the iPhone, that is,

$$
A=\{5,6,7, X\}
$$

Define two binary operations . and $*$ on $A$ as the following Cayley tables:

Then $A=(A, \cdot, *, X)$ is an $f$-UP-semigroup. Let $\left(\widetilde{\mathrm{F}}_{1}, E_{1}\right)$ and $\left(\widetilde{\mathrm{F}}_{2}, E_{2}\right)$ be two fuzzy soft sets over $A$ where

$$
E_{1}:=\{\text { price, beauty, specifications }\} \text { and } E_{2}:=\{\text { price, stability }\}
$$

with $\widetilde{\mathrm{F}}_{1}[$ price $], \widetilde{\mathrm{F}}_{1}\left[\right.$ beauty], $\widetilde{\mathrm{F}}_{1}[$ specifications $], \widetilde{\mathrm{F}}_{2}[$ price $]$, and $\widetilde{\mathrm{F}}_{2}[$ stability $]$ are fuzzy sets in A defined as follows:

\begin{tabular}{ccccc}
$\widetilde{\mathrm{F}}_{1}$ & $X$ & 7 & 6 & 5 \\
\hline price & 0.9 & 0.7 & 0.9 & 0.2 \\
beauty & 1 & 0.8 & 0.3 & 0.2 \\
specifications & 0.6 & 0.5 & 0.3 & 0.4 \\
\hline
\end{tabular}

\begin{tabular}{ccccc}
$\widetilde{\mathrm{F}}_{2}$ & $X$ & 7 & 6 & 5 \\
\hline price & 0.9 & 0.3 & 0.2 & 0.8 \\
stability & 0.7 & 0.2 & 0.5 & 0.2 \\
\hline
\end{tabular}


Then $\left(\widetilde{\mathrm{F}}_{1}, E_{1}\right)$ and $\left(\widetilde{\mathrm{F}}_{2}, E_{2}\right)$ are two fuzzy soft $U P_{\mathrm{s}}$-subalgebras of $A$. Since (price, price) $\in E_{1} \times E_{2}$, we have

$$
\begin{aligned}
\left(\mathrm{f}_{\widetilde{\mathrm{F}}_{1}[\text { price }] \cup \widetilde{\mathrm{F}}_{2}[\text { price }]}\right)(5 * 6) & =\left(\mathrm{f}_{\widetilde{\mathrm{F}}_{1}[\text { price }] \cup \widetilde{\mathrm{F}}_{2}[\text { price }]}\right)(7) \\
& =0.7 \\
& \ngtr 0.8 \\
& =\min \{0.8,0.9\} \\
& =\min \left\{\left(\mathrm{f}_{\widetilde{\mathrm{F}}_{1}[\text { price }] \cup \widetilde{\mathrm{F}}_{2}[\text { price }]}\right)(5),\left(\mathrm{f}_{\widetilde{\mathrm{F}}_{1}[\text { price }] \cup \widetilde{\mathrm{F}}_{2}[\text { price }]}\right)(6)\right\} .
\end{aligned}
$$

Thus $\widetilde{\mathrm{F}}_{1}[$ price $] \cup \widetilde{\mathrm{F}}_{2}[$ price $]$ is not a fuzzy UP $P_{\mathrm{s}}$-subalgebra of $A$, that is, $\left(\widetilde{\mathrm{F}}_{1}, E_{1}\right) \cup\left(\widetilde{\mathrm{F}}_{2}, E_{2}\right)$ is not a (price, price)fuzzy soft UP $P_{\mathrm{s}}$-subalgebra of $A$. Hence, $\left(\widetilde{\mathrm{F}}_{1}, E_{1}\right) \cup\left(\widetilde{\mathrm{F}}_{2}, E_{2}\right)$ is not a fuzzy soft UP $\mathrm{s}_{\mathrm{s}}$-subalgebra of $A$. Moreover, $\left(\widetilde{\mathrm{F}}_{1}, E_{1}\right) \vee\left(\widetilde{\mathrm{F}}_{2}, E_{2}\right)$ is not a fuzzy soft UP $\mathrm{s}$-subalgebra of $A$.

We can apply those examples in [16] to check that the OR of two fuzzy soft $\mathrm{UP}_{\mathrm{i}}$-subalgebras (resp., fuzzy soft near $\mathrm{UP}_{\mathrm{s}}$-filters, fuzzy soft $\mathrm{UP}_{\mathrm{s}}$-filters, fuzzy soft $\mathrm{UP}_{\mathrm{i}}$-filters, fuzzy soft $\mathrm{UP}_{\mathrm{s}}$-ideals, fuzzy soft $\mathrm{UP}_{\mathrm{i}}$-ideals) of $A$ is not fuzzy soft $\mathrm{UP}_{\mathrm{i}}$-subalgebra (resp., fuzzy soft near $\mathrm{UP}_{\mathrm{s}}$-filter, fuzzy soft $\mathrm{UP}_{\mathrm{s}}$-filter, fuzzy soft $\mathrm{UP}_{\mathrm{i}}$-filter, fuzzy soft $\mathrm{UP}_{\mathrm{s}}$-ideal, fuzzy soft $\mathrm{UP}_{\mathrm{i}}$-ideal).

We prove that certain distributive laws hold in fuzzy soft set theory with respect to the restricted union, the union, the intersection, and the extended intersection on any fuzzy soft sets.

Theorem 2.7. Let $\left(\widetilde{\mathrm{F}}_{i}, E_{i}\right)$ and $(\widetilde{\mathrm{F}}, E)$ be fuzzy soft sets over a common universe $U$ where $I$ is a nonempty set. Then the following properties hold:

(1) $(\widetilde{\mathrm{F}}, E) \cap\left(\bigcup_{i \in I}\left(\widetilde{\mathrm{F}}_{i}, E_{i}\right)\right)=\bigcup_{i \in I}\left((\widetilde{\mathrm{F}}, E) \cap\left(\widetilde{\mathrm{F}}_{i}, E_{i}\right)\right)$,

(2) $\left(\bigcup_{i \in I}\left(\widetilde{\mathrm{F}}_{i}, E_{i}\right)\right)$ ก $(\widetilde{\mathrm{F}}, E)=\bigcup_{i \in I}\left(\left(\widetilde{\mathrm{F}}_{i}, E_{i}\right)\right.$ ก $\left.(\widetilde{\mathrm{F}}, E)\right)$,

(3) $(\widetilde{\mathrm{F}}, E) \uplus\left(\bigcap_{i \in I}\left(\widetilde{\mathrm{F}}_{i}, E_{i}\right)\right)=\bigcap_{i \in I}\left((\widetilde{\mathrm{F}}, E) \uplus\left(\widetilde{\mathrm{F}}_{i}, E_{i}\right)\right)$,

(4) $\left.\left(\bigcap_{i \in I}\left(\widetilde{\mathrm{F}}_{i}, E_{i}\right)\right) \uplus(\widetilde{\mathrm{F}}, E)=\left(\widetilde{\mathrm{F}}_{i}, E_{i}\right)\right) \uplus \bigcap_{i \in I}((\widetilde{\mathrm{F}}, E)$,

(5) $(\widetilde{\mathrm{F}}, E) \cap\left(\bigcup_{i \in I}\left(\widetilde{\mathrm{F}}_{i}, E_{i}\right)\right)=\bigcup_{i \in I}\left((\widetilde{\mathrm{F}}, E) \cap\left(\widetilde{\mathrm{F}}_{i}, E_{i}\right)\right)$,

(6) $\left(\bigcup_{i \in I}\left(\widetilde{\mathrm{F}}_{i}, E_{i}\right)\right) \cap(\widetilde{\mathrm{F}}, E)=\bigcup_{i \in I}\left(\left(\widetilde{\mathrm{F}}_{i}, E_{i}\right) \cap(\widetilde{\mathrm{F}}, E)\right)$,

(7) $(\widetilde{\mathrm{F}}, E) \cup\left(\bigcap_{i \in I}\left(\widetilde{\mathrm{F}}_{i}, E_{i}\right)\right)=\bigcap_{i \in I}\left((\widetilde{\mathrm{F}}, E) \cup\left(\widetilde{\mathrm{F}}_{i}, E_{i}\right)\right)$,

(8) $\left(\bigcap_{i \in I}\left(\widetilde{\mathrm{F}}_{i}, E_{i}\right)\right) \cup(\widetilde{\mathrm{F}}, E)=\bigcap_{i \in I}\left(\left(\widetilde{\mathrm{F}}_{i}, E_{i}\right) \cup(\widetilde{\mathrm{F}}, E)\right)$,

(9) $(\widetilde{\mathrm{F}}, E)$ ก $\left(\bigcup_{i \in I}\left(\widetilde{\mathrm{F}}_{i}, E_{i}\right)\right)=\bigcup_{i \in I}\left((\widetilde{\mathrm{F}}, E)\right.$ ก $\left.\left(\widetilde{\mathrm{F}}_{i}, E_{i}\right)\right)$,

(10) (Uூ $\left.{ }_{i \in I}\left(\widetilde{\mathrm{F}}_{i}, E_{i}\right)\right)$ ก $(\widetilde{\mathrm{F}}, E)=\bigcup_{i \in I}\left(\left(\widetilde{\mathrm{F}}_{i}, E_{i}\right)\right.$ ก $\left.(\widetilde{\mathrm{F}}, E)\right)$,

(11) $(\widetilde{\mathrm{F}}, E) \uplus\left(\bigcap_{i \in I}\left(\widetilde{\mathrm{F}}_{i}, E_{i}\right)\right)=\bigcap_{i \in I}\left((\widetilde{\mathrm{F}}, E) \uplus\left(\widetilde{\mathrm{F}}_{i}, E_{i}\right)\right)$, and

(12) $\left(\bigcap_{i \in I}\left(\widetilde{\mathrm{F}}_{i}, E_{i}\right)\right) \uplus(\widetilde{\mathrm{F}}, E)=\bigcap_{i \in I}\left(\left(\widetilde{\mathrm{F}}_{i}, E_{i}\right) \uplus(\widetilde{\mathrm{F}}, E)\right)$.

Proof. (1) First, we investigate left hand side of the equality. Suppose that $\bigcup_{i \in I}\left(\widetilde{\mathrm{F}}_{i}, E_{i}\right)=\left(\widetilde{\mathrm{G}}, E^{U}\right)$ is the union of $\left(\widetilde{\mathrm{F}}_{i}, E_{i}\right)$ for all $i \in I$. Then $E^{U}=\bigcup_{i \in I} E_{i}$ and for any $e \in E^{U}, \widetilde{\mathrm{G}}[e]=\bigcup_{j \in J} \widetilde{\mathrm{F}}_{j}[e]$ with 
$e \in \bigcap_{j \in J} E_{j}-\bigcup_{k \in I-J} E_{k}$ where $\emptyset \neq J \subseteq I$. Thus $(\widetilde{\mathrm{F}}, E) \cap\left(\bigcup_{i \in I}\left(\widetilde{\mathrm{F}}_{i}, E_{i}\right)\right)=(\widetilde{\mathrm{F}}, E) \cap\left(\widetilde{\mathrm{G}}, E^{U}\right)=\left(\widetilde{\mathrm{H}}, E^{U I}\right)$. For any $e \in E^{U I}=E \cap E^{U} \neq \emptyset, \widetilde{\mathrm{H}}[e]=\widetilde{\mathrm{F}}[e] \cap \widetilde{\mathrm{G}}[e]$ where $E \cap E^{U}=E \cap\left(\bigcup_{i \in I} E_{i}\right)=\bigcup_{i \in I}\left(E \cap E_{i}\right)$. By considering $\widetilde{\mathrm{G}}$ as piecewise defined function, we have $\widetilde{\mathrm{H}}[e]=\widetilde{\mathrm{F}}[e] \cap\left(\bigcup_{j \in J} \widetilde{\mathrm{F}}_{j}[e]\right)$ with $e \in \bigcap_{j \in J}\left(E \cap E_{j}\right)-\bigcup_{k \in I-J}\left(E \cap E_{k}\right)$ where $\emptyset \neq J \subseteq I$.

Consider the right hand side of the equality. Suppose that $(\widetilde{\mathrm{F}}, E) \cap\left(\widetilde{\mathrm{F}}_{i}, E_{i}\right)=\left(\widetilde{\mathrm{I}}_{i}, E_{i}^{I}\right)$ is the intersection of $(\widetilde{\mathrm{F}}, E)$ and $\left(\widetilde{\mathrm{F}}_{i}, E_{i}\right)$ for all $i \in I$. Then $E_{i}^{I}=E \cap E_{i} \neq \emptyset$ and for any $e \in E_{i}^{I}, \widetilde{\mathrm{I}}_{i}[e]=\widetilde{\mathrm{F}}[e] \cap \widetilde{\mathrm{F}}_{i}[e]$. Now, $\bigcup_{i \in I}\left((\widetilde{\mathrm{F}}, E) \cap\left(\widetilde{\mathrm{F}}_{i}, E_{i}\right)\right)=\bigcup_{i \in I}\left(\widetilde{\mathrm{I}}_{i}, E_{i}^{I}\right)=\left(\widetilde{\mathrm{J}}, E^{I U}\right)$, where $E^{I U}=\bigcup_{i \in I} E_{i}^{I}=\bigcup_{i \in I}\left(E \cap E_{i}\right)$. For any $e \in E^{I U}, \widetilde{\mathrm{J}}[e]=\bigcup_{j \in J} \widetilde{\mathrm{I}}_{j}[e]$ with $e \in \bigcap_{j \in J} E_{j}^{I}-\bigcup_{k \in I-J} E_{k}^{I}$ where $\emptyset \neq J \subseteq I$. Considering $\widetilde{\mathrm{I}}_{i}$ as piecewise functions for all $i \in I$, we have $\widetilde{\mathrm{J}}[e]=\bigcup_{j \in J}\left(\widetilde{\mathrm{F}}[e] \cap \widetilde{\mathrm{F}}_{j}[e]\right)$ with $e \in \bigcap_{j \in J}\left(E \cap E_{j}\right)-\bigcup_{k \in I-J}\left(E \cap E_{k}\right)$ where $\emptyset \neq J \subseteq I$. By Theorem 1.1 (1), it is clear that $\widetilde{\mathrm{H}}$ and $\widetilde{\mathrm{J}}$ are same set-valued mapping. Hence, $(\widetilde{\mathrm{F}}, E)$ ก $\left(\bigcup_{i \in I}\left(\widetilde{\mathrm{F}}_{i}, E_{i}\right)\right)=\bigcup_{i \in I}\left((\widetilde{\mathrm{F}}, E)\right.$ ก $\left.\left(\widetilde{\mathrm{F}}_{i}, E_{i}\right)\right)$.

(2) By using techniques as in (1) and by Theorem 1.1 (2), then (2) can be derived.

(3) By using techniques as in (1) and by Theorem 1.1 (3), then (3) can be derived.

(4) By using techniques as in (1) and by Theorem 1.1 (4), then (4) can be derived.

(5) First, we investigate left hand side of the equality. Suppose that $\bigcup_{i \in I}\left(\widetilde{\mathrm{F}}_{i}, E_{i}\right)=\left(\widetilde{\mathrm{G}}, E^{R U}\right)$ is the restricted union of $\left(\widetilde{\mathrm{F}}_{i}, E_{i}\right)$ for all $i \in I$. Then $E^{R U}=\bigcap_{i \in I} E_{i} \neq \emptyset$ and for any $e \in E^{R U}, \widetilde{\mathrm{G}}[e]=\bigcup_{i \in I} \widetilde{\mathrm{F}}_{i}[e]$. Thus $(\widetilde{\mathrm{F}}, E) \cap\left(\bigcup_{i \in I}\left(\widetilde{\mathrm{F}}_{i}, E_{i}\right)\right)=(\widetilde{\mathrm{F}}, E) \cap\left(\widetilde{\mathrm{G}}, E^{R U}\right)=\left(\widetilde{\mathrm{H}}, E^{R U E I}\right)$. For any $e \in E^{R U E I}=E \cup E^{R U}$, we have

$$
\widetilde{\mathrm{H}}[e]= \begin{cases}\widetilde{\mathrm{F}}[e] & \text { if } e \in E \backslash E^{R U} \\ \widetilde{\mathrm{G}}[e] & \text { if } e \in E^{R U} \backslash E \\ \widetilde{\mathrm{F}}[e] \cap \widetilde{\mathrm{G}}[e] & \text { if } e \in E \cap E^{R U}\end{cases}
$$

By taking into account the definition of $\widetilde{\mathrm{G}}$ along with $\widetilde{\mathrm{H}}$, we can write

$$
\widetilde{\mathrm{H}}[e]= \begin{cases}\widetilde{\mathrm{F}}[e] & \text { if } e \in E \backslash\left(\bigcap_{i \in I} E_{i}\right) \\ \bigcup_{i \in I} \widetilde{\mathrm{F}}_{i}[e] & \text { if } e \in\left(\bigcap_{i \in I} E_{i}\right) \backslash E \\ \widetilde{\mathrm{F}}[e] \cap\left(\bigcup_{i \in I} \widetilde{\mathrm{F}}_{i}[e]\right) & \text { if } e \in E \cap\left(\bigcap_{i \in I} E_{i}\right) .\end{cases}
$$

Consider the right hand side of the equality. Suppose that $(\widetilde{\mathrm{F}}, E) \cap\left(\widetilde{\mathrm{F}}_{i}, E_{i}\right)=\left(\widetilde{\mathrm{I}}_{i}, E_{i}^{E I}\right)$ is the extended intersection of $(\widetilde{\mathrm{F}}, E)$ and $\left(\widetilde{\mathrm{F}}_{i}, E_{i}\right)$ for all $i \in I$. Then for any $e \in E_{i}^{E I}=E \cup E_{i}$, we have

$$
\widetilde{\mathrm{I}}_{i}[e]= \begin{cases}\widetilde{\mathrm{F}}[e] & \text { if } e \in E \backslash E_{i} \\ \widetilde{\mathrm{F}}_{i}[e] & \text { if } e \in E_{i} \backslash E \\ \widetilde{\mathrm{F}}[e] \cap \widetilde{\mathrm{F}}_{i}[e] & \text { if } e \in E \cap E_{i} .\end{cases}
$$

Now, $\left.\bigcup_{i \in I}\left((\widetilde{\mathrm{F}}, E) \cap\left(\widetilde{\mathrm{F}}_{i}, E_{i}\right)\right)=\bigcup_{i \in I} \widetilde{\mathrm{I}}_{i}, E_{i}^{E I}\right)=\left(\widetilde{\mathrm{J}}, E^{E I R U}\right)$ where $E^{E I R U}=\bigcap_{i \in I} E_{i}^{I}=\bigcap_{i \in I}\left(E \cup E_{i}\right)=$ $E \cup\left(\bigcap_{i \in I} E_{i}\right) \neq \emptyset$. For any $e \in E^{E I R U}, \widetilde{\mathrm{J}}[e]=\bigcup_{i \in I} \widetilde{\mathrm{I}}_{i}[e]$. By taking into account the properties of operations 
in set theory and considering $\widetilde{\mathrm{I}}_{i}$ as piecewise defined functions for all $i \in I$, we have

$$
\widetilde{\mathrm{J}}[e]= \begin{cases}\bigcup_{i \in I} \widetilde{\mathrm{F}}[e] & \text { if } e \in E \backslash\left(\bigcap_{i \in I} E_{i}\right) \\ \bigcup_{i \in I} \widetilde{\mathrm{F}}_{i}[e] & \text { if } e \in\left(\bigcap_{i \in I} E_{i}\right) \backslash E \\ \bigcup_{i \in I}\left(\widetilde{\mathrm{F}}[e] \cap \widetilde{\mathrm{F}}_{i}[e]\right) & \text { if } e \in E \cap\left(\bigcap_{i \in I} E_{i}\right) .\end{cases}
$$

And so

$$
\widetilde{\mathrm{J}}[e]= \begin{cases}\widetilde{\mathrm{F}}[e] & \text { if } e \in E \backslash\left(\bigcap_{i \in I} E_{i}\right) \\ \bigcup_{i \in I} \widetilde{\mathrm{F}}_{i}[e] & \text { if } e \in\left(\bigcap_{i \in I} E_{i}\right) \backslash E \\ \bigcup_{i \in I}\left(\widetilde{\mathrm{F}}[e] \cap \widetilde{\mathrm{F}}_{i}[e]\right) & \text { if } e \in E \cap\left(\bigcap_{i \in I} E_{i}\right) .\end{cases}
$$

By Theorem $1.1(1)$, it is clear that $\widetilde{\mathrm{H}}$ and $\widetilde{\mathrm{J}}$ are same set-valued mapping. Hence, $(\widetilde{\mathrm{F}}, E) \cap\left(\bigcup_{i \in I}\left(\widetilde{\mathrm{F}}_{i}, E_{i}\right)\right)=$ $\bigcup_{i \in I}\left((\widetilde{\mathrm{F}}, E) \cap\left(\widetilde{\mathrm{F}}_{i}, E_{i}\right)\right)$.

(6) By using techniques as in (5) and by Theorem 1.1 (2), then (6) can be derived.

(7) By using techniques as in (5) and by Theorem 1.1 (3), then (7) can be derived.

(8) By using techniques as in (5) and by Theorem 1.1 (4), then (8) can be derived.

(9) First, we investigate left hand side of the equality. Suppose that $\bigcup_{i \in I}\left(\widetilde{\mathrm{F}}_{i}, E_{i}\right)=\left(\widetilde{\mathrm{G}}, E^{R U}\right)$ is the restricted union of $\left(\widetilde{\mathrm{F}}_{i}, E_{i}\right)$ for all $i \in I$. Then $E^{R U}=\bigcap_{i \in I} E_{i} \neq \emptyset$ and for any $e \in E^{R U}, \widetilde{\mathrm{G}}[e]=\bigcup_{i \in I} \widetilde{\mathrm{F}}_{i}[e]$. Thus $(\widetilde{\mathrm{F}}, E) \cap\left(\bigcup_{i \in I}\left(\widetilde{\mathrm{F}}_{i}, E_{i}\right)\right)=(\widetilde{\mathrm{F}}, E) \cap\left(\widetilde{\mathrm{G}}, E^{R U}\right)=\left(\widetilde{\mathrm{H}}, E^{R U I}\right)$. For any $e \in E^{R U I}=E \cap E^{R U}=E \cap$ $\left(\bigcap_{i \in I} E_{i}\right) \neq \emptyset$, we have $\widetilde{\mathrm{H}}[e]=\widetilde{\mathrm{F}}[e] \cap \widetilde{\mathrm{G}}[e]=\widetilde{\mathrm{F}}[e] \cap\left(\bigcup_{i \in I} \widetilde{\mathrm{F}}_{i}[e]\right)$.

Consider the right hand side of the equality. Suppose that $(\widetilde{\mathrm{F}}, E) \cap\left(\widetilde{\mathrm{F}}_{i}, E_{i}\right)=\left(\widetilde{\mathrm{I}}_{i}, E_{i}^{I}\right)$ is the intersection of $(\widetilde{\mathrm{F}}, E)$ and $\left(\widetilde{\mathrm{F}}_{i}, E_{i}\right)$ for all $i \in I$. Then $E_{i}^{I}=E \cap E_{i} \neq \emptyset$ and for any $e \in E_{i}^{I}, \widetilde{\mathrm{I}}_{i}[e]=\widetilde{\mathrm{F}}[e] \cap \widetilde{\mathrm{F}}_{i}[e]$. Now, $\bigcup_{i \in I}\left((\widetilde{\mathrm{F}}, E)\right.$ ก $\left.\left(\widetilde{\mathrm{F}}_{i}, E_{i}\right)\right)=\bigcup_{i \in I}\left(\widetilde{\mathrm{I}}_{i}, E_{i}^{I}\right)=\left(\widetilde{\mathrm{J}}, E^{I R U}\right)$, where $E^{I R U}=\bigcap_{i \in I} E_{i}^{I}=\bigcap_{i \in I}\left(E \cap E_{i}\right) \neq \emptyset$.

For any $e \in E^{I R U}, \widetilde{J}[e]=\bigcup_{j \in J} \widetilde{\mathrm{I}}_{j}[e]=\bigcup_{j \in J}\left(\widetilde{\mathrm{F}}[e] \cap \widetilde{\mathrm{F}}_{i}[e]\right)$. Since $\bigcap_{i \in I}\left(E \cap E_{i}\right)=E \cap\left(\bigcap_{i \in I} E_{i}\right)$, we have $E^{I R U}=E^{R U I}$. By Theorem $1.1(1)$, it is clear that $\widetilde{\mathrm{H}}$ and $\widetilde{\mathrm{J}}$ are same set-valued mapping. Hence, $(\widetilde{\mathrm{F}}, E) \cap\left(\bigcup_{i \in I}\left(\widetilde{\mathrm{F}}_{i}, E_{i}\right)\right)=\bigcup_{i \in I}\left((\widetilde{\mathrm{F}}, E) \cap\left(\widetilde{\mathrm{F}}_{i}, E_{i}\right)\right)$.

(10) By using techniques as in (9) and by Theorem 1.1 (2), then (10) can be derived.

(11) By using techniques as in (9) and by Theorem 1.1 (3), then (11) can be derived.

(12) By using techniques as in (9) and by Theorem 1.1 (4), then (12) can be derived.

\section{ACKNOWLEDGMENT}

The authors would also like to thank the anonymous referee for giving many helpful suggestion on the revision of present paper.

\section{REFERENCES}

[1] B. Ahmad and A. Kharal, On fuzzy soft sets, Adv. Fuzzy Syst. 2009 (2009), Article ID 586507.

[2] M. A. Ansari, A. Haidar, and A. N. A. Koam, On a graph associated to UP-algebras, Math. Comput. Appl. 23 (2018), no. 4,61 . 
[3] N. Dokkhamdang, A. Kesorn, and A. Iampan, Generalized fuzzy sets in UP-algebras, Ann. Fuzzy Math. Inform. 16 (2018), no. $2,171-190$.

[4] T. Guntasow, S. Sajak, A. Jomkham, and A. Iampan, Fuzzy translations of a fuzzy set in UP-algebras, J. Indones. Math. Soc. 23 (2017), no. 2, 1-19.

[5] A. Iampan, A new branch of the logical algebra: UP-algebras, J. Algebra Relat. Top. 5 (2017), no. 1, $35-54$.

[6] A. Iampan, Introducing fully UP-semigroups, Discuss. Math., Gen. Algebra Appl. 38 (2018), no. 2, $297-306$.

[7] Y. B. Jun, S. M. Hong, and E. H. Roh, BCI-semigroups, Honam Math. J. 15 (1993), no. 1, 59-64.

[8] Y. B. Jun, K. J. Lee, and C. H. Park, Fuzzy soft set theory applied to BCK/BCI-algebras, Comput. Math. Appl. 59 (2010), $3180-3192$.

[9] K. H. Lee, First course on fuzzy theory and applications, Springer-Verlag Berlin Heidelberg, Republic of South Korea, 2005 .

[10] P.K. Maji, R. Biswas, and A.R. Roy, Fuzzy soft sets, J. Fuzzy Math. 9 (2001), no. 3, 589-602.

[11] D. Molodtsov, Soft set theory-first results, Comput. Math. Appl. 37 (1999), 19-31.

[12] C. Prabpayak and U. Leerawat, On ideals and congruences in KU-algebras, Sci. Magna 5 (2009), no. $1,54-57$.

[13] A. Rehman, S. Abdullah, M. Aslam, and M. S. Kamran, A study on fuzzy soft set and its operations, Ann. Fuzzy Math. Inform. 6 (2013), no. 2, 339-362.

[14] A. Rosenfeld, Fuuzy groups, J. Math, Anal. Appl. 35 (1971), 512-517.

[15] A. Satirad and A. Iampan, Fuzzy sets in fully UP-semigroups, Manuscript accepted for publication in Ital. J. Pure Appl. Math., July 2018.

[16] A. Satirad and A. Iampan, Fuzzy soft sets over fully UP-semigroups, Eur. J. Pure Appl. Math. 12 (2019), no. 2, $294-331$.

[17] A. Satirad, P. Mosrijai, and A. Iampan, Formulas for finding UP-algebras, Int. J. Math. Comput. Sci. 14 (2019), no. 2, 403-409.

[18] A. Satirad and A. Iampan, Generalized power UP-algebras, Int. J. Math. Comput. Sci. 14 (2019), no. 1, $17-25$.

[19] J. Somjanta, N. Thuekaew, P. Kumpeangkeaw, and A. Iampan, Fuzzy sets in UP-algebras, Ann. Fuzzy Math. Inform. 12 (2016), no. 6, 739-756.

[20] L. A. Zadeh, Fuzzy sets, Inf. Control, 8 (1965), 338-353. 